\title{
New Source of Dense, Cryogenic Positron Plasmas
}

L. V. Jørgensen, ${ }^{1, *}$ M. Amoretti, ${ }^{2}$ G. Bonomi, ${ }^{3}$ P. D. Bowe, ${ }^{1}$ C. Canali, ${ }^{2,4}$ C. Carraro, ${ }^{2,4}$ C. L. Cesar, ${ }^{5}$ M. Charlton, ${ }^{1}$ M. Doser, ${ }^{3}$ A. Fontana, ${ }^{6,7}$ M. C. Fujiwara, ${ }^{8,9}$ R. Funakoshi, ${ }^{10}$ P. Genova, ${ }^{6,7}$ J. S. Hangst, ${ }^{11}$ R. S. Hayano, ${ }^{10}$ A. Kellerbauer,${ }^{3}$ V. Lagomarsino, ${ }^{2,4}$ R. Landua, ${ }^{3}$ E. Lodi Rizzini, ${ }^{12}$ M. Macrì ${ }^{2}$ N. Madsen, ${ }^{11}$ D. Mitchard, ${ }^{1}$ P. Montagna,,${ }^{6,7}$ A. Rotondi, ${ }^{6,7}$ G. Testera, ${ }^{2}$ A. Variola, ${ }^{2}$ L. Venturelli, ${ }^{12}$ D. P. van der Werf, ${ }^{1}$ and Y. Yamazaki ${ }^{9}$

(ATHENA Collaboration $)^{\dagger}$

${ }^{1}$ Department of Physics, University of Wales Swansea, Swansea SA2 8PP, United Kingdom

${ }^{2}$ Istituto Nazionale di Fisica Nucleare, Sezione di Genova, 16146 Genova, Italy

${ }^{3}$ PH Department, CERN, 1211 Geneva 23, Switzerland

${ }^{4}$ Dipartimento di Fisica, Universita di Genova, 16146 Genova, Italy

${ }^{5}$ Instituto de Fisica, Universidade do Brasil, Cx Postal 68528, Rio de Janeiro 21941-972, Brazil

${ }^{6}$ Istituto Nazionale di Fisica Nucleare, Sezione di Pavia, 27100 Pavia, Italy

${ }^{7}$ Dipartimento di Fisica Nucleare e Teorica, Universita di Pavia, 27100 Pavia, Italy

${ }^{8}$ TRIUMF, 4004 Wesbrook Mall Vancouver, BC V6T 2A3, Canada

${ }^{9}$ Atomic Physics Laboratory, RIKEN, Saitama 351-0198, Japan

${ }^{10}$ Department of Physics, University of Tokyo, Tokyo 153-8902, Japan

${ }^{11}$ Department of Physics and Astronomy, University of Aarhus, 8000 Aarhus C, Denmark

${ }^{12}$ Dipartimento di Chimica e Fisica per l'Ingegneria e per i Materiali, Universita di Brescia, and INFN gruppo Collegato di Brescia, 25123 Brescia, Italy

(Received 4 May 2004; revised manuscript received 22 April 2005; published 7 July 2005)

\begin{abstract}
We have developed a new method, based on the ballistic transfer of preaccumulated plasmas, to obtain large and dense positron plasmas in a cryogenic environment. The method involves transferring plasmas emanating from a region with a low magnetic field $(0.14 \mathrm{~T})$ and relatively high pressure $\left(10^{-9} \mathrm{mbar}\right)$ into a $15 \mathrm{~K}$ Penning-Malmberg trap immersed in a $3 \mathrm{~T}$ magnetic field with a base pressure better than $10^{-13}$ mbar. The achieved positron accumulation rate in the high field cryogenic trap is more than one and a half orders of magnitude higher than the previous most efficient UHV compatible scheme. Subsequent stacking resulted in a plasma containing more than $1.2 \times 10^{9}$ positrons, which is a factor 4 higher than previously reported. Using a rotating wall electric field, plasmas containing about $20 \times 10^{6}$ positrons were compressed to a density of $2.6 \times 10^{10} \mathrm{~cm}^{-3}$. This is a factor of 6 improvement over earlier measurements.
\end{abstract}

DOI: 10.1103/PhysRevLett.95.025002

PACS numbers: 52.27.Jt, 36.10.- $-\mathrm{k}$

The development of laboratory positron plasmas over the last 15 years has dramatically enhanced capabilities for basic antimatter-matter physics research. These plasmas are used, for instance, to produce cold beams to study positron interactions with atoms and molecules in unprecedented detail ([1] and references therein). Intense positron pulses are to be used in the formation and study of positronium molecules $\left(\mathrm{Ps}_{2}\right)$ and in attempts to make a positronium Bose-Einstein condensate [2], as well as in studies of Rydberg state positronium [3] and as a means of efficient cooling of highly charged ions for high resolution spectroscopy and mass measurements [4]. These applications are facilitated by large numbers of positrons as well as a high density of the positron plasma. Such experiments have led to increased interest in extending techniques for accumulating and storing large, high density positron plasmas in a cryogenic environment $[5,6]$. It is expected that many of these experiments will need to accumulate positrons externally and subsequently transfer them to the experimental region.
Recently, antiprotons and positron plasmas were used to produce cold antihydrogen atoms, first by the ATHENA Collaboration [7] and subsequently by ATRAP [8] at the CERN Antiproton Decelerator (AD). A large and dense positron plasma collected on the short time scale of the $\mathrm{AD}$ cycle $(\sim 100 \mathrm{~s})$ was essential to the success of the ATHENA experiment. Indeed, this is one of the features that sets the two experiments apart. In both antihydrogen experiments, atoms are formed by mixing antiprotons and positrons in a nested Penning trap [9]. The relevant reaction mechanisms are radiative and three-body recombination ([10] and references therein), the reaction rates in the simplest approximation being proportional to the positron density as $n$ and $n^{2}$, respectively. Three-body recombination is expected to be the dominant process in the experiments reported so far. This process predominantly creates antihydrogen atoms in high Rydberg states that subsequently may need to collisionally deexcite if they are to avoid field ionization. A high density and large number of positrons should greatly enhance this process, leading to 
more deeply bound and thus experimentally more useful antihydrogen. Large and dense positron plasmas will become even more important if plans to decelerate the antiprotons from the AD even further are realized. This could lead to up to 2 orders of magnitude more antiprotons trapped per AD shot.

In order to rapidly acquire and manipulate large numbers of positrons ready to mix with antiprotons in the ATHENA experiment, we have constructed an external accumulator utilizing nitrogen as a buffer gas [11,12]. This device is interfaced to a Penning-Malmberg trap housing the main mixing region. In this Letter, we report on the relevant physics of the trapping and retrapping developed to manipulate the positron plasma and transfer it to a cryogenic, extreme high-vacuum environment, together with the results obtained.

The ATHENA antihydrogen apparatus [13] consists of four main parts: a positron accumulator, an antiproton catching trap, a mixing trap, and an annihilation detector. The accumulator (gas pressure $10^{-6}-10^{-9} \mathrm{mbar}$ ) and the mixing trap (better than $10^{-13}$ mbar, electrode radius $12.5 \mathrm{~mm}$ ) are connected by a transfer section consisting of a vacuum separation valve, a pumping restriction, a number of transfer electrodes, and a pulsed solenoid capable of producing a field of $1 \mathrm{~T}$. The transfer magnet is necessary to collimate the positrons in the pumping restriction.

The number of particles caught in the mixing region can be detected by dumping them onto a Faraday cup. This number can be compared with measurements on a similar Faraday cup that can be inserted at the end of the positron accumulator allowing optimization of the transfer parameters. In order to increase the density of the plasma inside the $3 \mathrm{~T}$ solenoid, it can be compressed by employing a rotating wall (RW) electric field $[14,15]$. A nondestructive plasma diagnostic technique has recently been developed using electrostatic mode analysis $[16,17]$ facilitating realtime monitoring of the plasma.

Positrons are accumulated in a relatively low $(0.14 \mathrm{~T})$ magnetic field in the main accumulation region [12]. During accumulation a RW electric field with a frequency of $500 \mathrm{kHz}$ and an amplitude of $0.4 \mathrm{~V}$ is applied in order to compress the plasma and achieve a larger accumulated total number. A $200 \mathrm{~s}$ accumulation yields a plasma of about $120-150 \times 10^{6}$ positrons with a diameter of $3-$ $4 \mathrm{~mm}$. The buffer gas is then pumped out to better than $5 \times$ $10^{-9}$ mbar and the vacuum separation valve is opened and the transfer magnet energized.

The positrons are released from the accumulator by lowering the exit electrode (see Fig. 1) from 180 to $0 \mathrm{~V}$ in $35 \mathrm{~ns}$. This corresponds to a fall time of 1 ns over the plasma space charge of about $5 \mathrm{~V}$. The base of the positron accumulator is kept at $25 \mathrm{~V}$, while all transfer and mixing trap electrodes are kept at ground during the transfers, thus accelerating the positrons to a kinetic energy of $25 \mathrm{eV}$ as they leave the accumulator. The positrons are retrapped, initially in the entire length of the mixing section, by closing the electrodes in the transfer section a time $\Delta T$

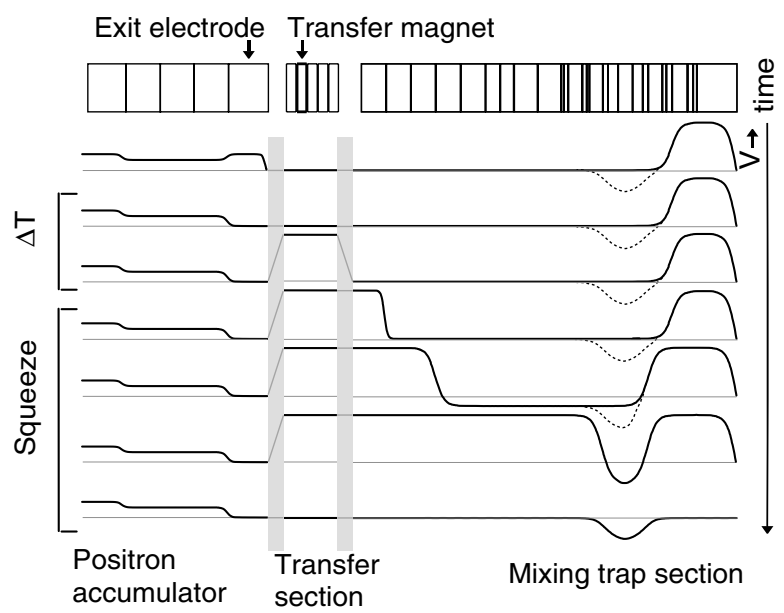

FIG. 1. The axial potentials in the traps during positron transfers. Note that the time steps between the subsequent potential distributions are not the same. The dashed lines represent the potentials when stacking subsequent positron shots. All potentials are to scale (maximum, $140 \mathrm{~V}$; minimum, $-50 \mathrm{~V}$ ).

after the exit electrode in the positron accumulator opened. The positron plasma expands as it passes through the low magnetic field between the solenoids (minimum field $\sim 0.02 \mathrm{~T}$ ) before arriving in the $3 \mathrm{~T}$ field of the mixing trap. Because of aperture restrictions as the positrons pass through the varying magnetic fields of the transfer region, only $56 \%$ of the positrons in the accumulator are transported to the mixing trap and are available for retrapping.

Figure 2 shows the fraction of retrapped positrons as $\Delta T$ was varied. The data were obtained by dumping the positrons on the Faraday cup $50 \mathrm{~ms}$ after retrapping and show that it is possible to retrap all the positrons that reach the mixing trap. The additional peaks are due to positrons bouncing back and forth between the positron accumulator and the mixing trap, indicating that the transport is indeed ballistic.

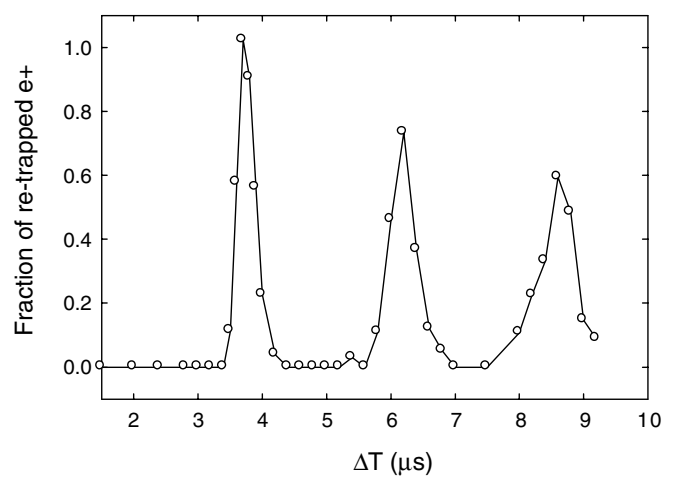

FIG. 2. The fraction of retrapped positrons as a function of the time, $\Delta T$, between opening the exit electrode and closing the transfer section. The data are normalized to the number of positrons that reach the Faraday cup without trapping. $\Delta T$ is an absolute scale and includes a delay in the electronics chain of about $2.5 \mu \mathrm{s}$. The lines are only to guide the eye. 
Once the positrons are in the main magnet, they cool to the ambient temperature of $15 \mathrm{~K}$ by emission of synchrotron radiation in the $3 \mathrm{~T}$ field with a characteristic cooling time of $0.5 \mathrm{~s}$. After the initial retrapping the positrons are subsequently squeezed (Fig. 1) into the harmonic region of the mixing trap. The timing of each step in the squeeze was optimized by observing the loss of positrons in the trap using the 192 CsI diode detectors of the main ATHENA detector [18]. The squeeze caused adiabatic heating of the plasma, which resulted in some positrons being lost by passing over the potential barriers at the ends. This effect was minimized by gradually lowering the bottom potential of the trap during the squeeze. In total, the squeeze took about $25 \mathrm{~s}$ and gave rise to additional losses up to $35 \%$, giving an overall efficiency of around $36 \%$; i.e., we are able transfer about $45-55 \times 10^{6}$ positrons every $200 \mathrm{~s}$. The resulting positron plasma in the harmonic mixing trap had a typical density of $8.0 \times 10^{8} \mathrm{~cm}^{-3}$ and a diameter of about $2 \mathrm{~mm}$. The transfer efficiency reported here corresponds to an overall accumulation rate into the cryogenic UHV region of $7.6 \pm 1.4 \times 10^{3} e^{+} / \mathrm{s} / \mathrm{mCi}$ of ${ }^{22} \mathrm{Na}$. This is superior to other UHV compatible positron accumulation schemes such as an electron plasma based approach [19], field ionization of Rydberg positronium [20], and resistive cooling [21], which have accumulation rates of $3.6 \times 10^{2}, 1.1 \times 10^{1}$, and $3.3 \times 10^{-2} e^{+} / \mathrm{s} / \mathrm{mCi}$, respectively.

An alternative approach to the trap-and-squeeze technique has also been tested following a shortening of the physical length of the mixing trap. Here it was necessary to shape the potentials of the trap to slow down the positrons after they had entered the trap, leaving more time to close the gate electrode behind them. The retrapping efficiency was highest when we no longer actively squeezed the positrons into the final well but allowed them to self-cool into an ever shorter trap, finally residing in the central harmonic well. This was done by applying a slope to the potential in the mixing trap. The self-cooling time used was about $20 \mathrm{~s}$, allowing the positrons ample time to reach the bottom of the central well. After optimization this configuration yielded an overall retrapping efficiency of about $45 \%$.

For some experiments with antihydrogen, it is useful to have significantly larger numbers of positrons available. To achieve this we have stacked subsequent shots of positrons from the positron accumulator. Positrons have been stacked before [22] even involving trap-to-trap transfer through low nonhomogeneous magnetic fields [23], but the field variations were much smaller than in our case and did not entail the eventual accumulation in a high-field, cryogenic, extreme high-vacuum environment. Stacking of a number of positron shots shows a linear behavior until the space charge limit of the $40 \mathrm{~V}$ well has been reached after about 4 shots, obtaining a plasma containing about $200 \times$ $10^{6}$ positrons. By gradually deepening the harmonic well to $140 \mathrm{~V}$ as more positrons are transferred, it is possible to reach much higher numbers. Figure 3 shows the number of positrons in the mixing trap as a function of the number of shots stacked. To ascertain the reproducibility of the data, 25 stack experiments were done at the start and end of the measurement, the lower point at 25 stacks being the final measurement. The results show a near-linear behavior up to amplifier saturation with about $47 \times 10^{6}$ positrons per stack. The largest plasma contained at least $1.2 \times 10^{9}$ positrons, which is a factor of 4 more than the largest positron plasma reported previously [24]. The long lifetime of the positron plasma in the cryogenic vacuum of the mixing trap ( $\gg 1 \mathrm{~h}$ ) makes it unlikely that losses due to the lifetime of the plasma contributed to the saturation level, although the stacking of 40 shots takes about $2.5 \mathrm{~h}$.

We have employed the RW technique in the mixing trap to allow us to control the shape and density of the positron plasma during antihydrogen formation. We use a four-way segmented electrode with a length of $10 \mathrm{~mm}$ directly adjacent to the central electrode (length $15 \mathrm{~mm}$ ) to apply an $m_{\theta}=1$ azimuthal perturbation to the positron plasma. Extensive surveys were made to optimize the performance in terms of frequency, amplitude, sweep, and duration. During the experiments the plasma parameters were monitored using the mode analysis system; in particular, the $(1,0)$ dipole and $(2,0)$ quadrupole frequencies [17] were used to obtain the plasma density, aspect ratio, and length. The positron number was cross-checked by dumping the positrons on a Faraday cup at the end of each measurement. Figure 4 shows the result of such a manipulation. The measurement is divided into three time regions: before, during, and after the application of the RW. In many cases the modes signal during the application of the RW is lost or very weak, and therefore unreliable. Furthermore, applying the RW leads to plasma heating, and as the quadropole mode frequency is sensitive to both compression and plasma temperature, the difficulty of disentangling these two effects renders data obtained during RW-on periods unusable. The plasma heating can be seen from the rapid decrease in the quadrupole frequency between the first and second measurement point after the RW is switched off. This is attributed to synchrotron cooling of the plasma in

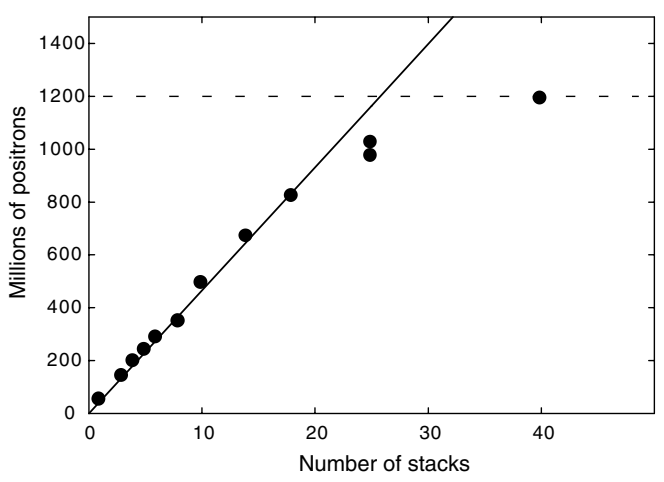

FIG. 3. The number of positrons versus the number of stacks. The solid line is a fit to the data for the first 20 stacks and yields a stacking rate of $47 \times 10^{6}$ positrons per stack. The dashed line indicates the Faraday cup amplifier saturation level. 

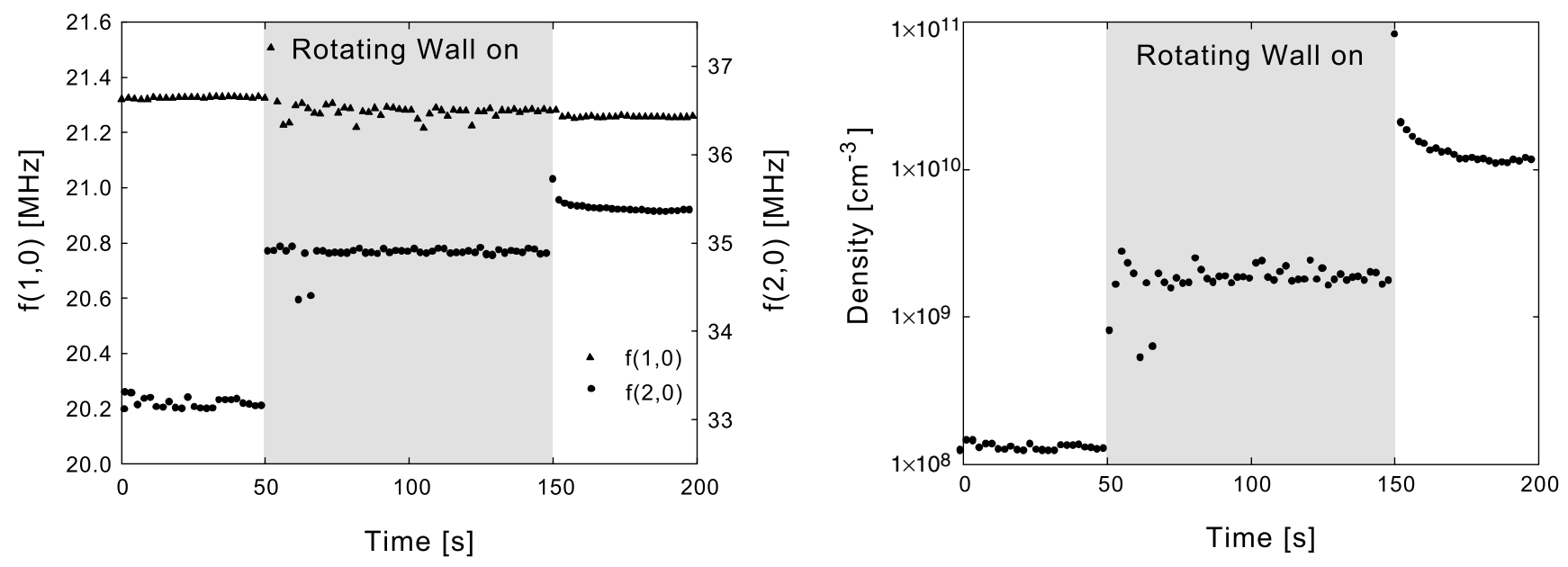

FIG. 4. Typical example of the two mode frequencies and the density when applying the RW in compression mode. For these measurements a frequency of $15.0 \mathrm{MHz}$ was used with an amplitude of $3 \mathrm{~V}$. The plasmas used typically contained $20 \times 10^{6}$ positrons. Only the data obtained before and after the RW-on time period are reliable, as the RW affects the modes measurement.

the $3 \mathrm{~T}$ field. By using the independent measure of the number of positrons in the plasma from the dump at the end, it is possible to estimate the size of the heating effect from the RW. We find that the heating typically raised the temperature by $\sim 0.2 \mathrm{eV}$. For a plasma containing $20 \times$ $10^{6}$ positrons with an initial density of $1.2 \times 10^{8} \mathrm{~cm}^{-3}$, an aspect ratio of 5.7, and a radius of $1.9 \mathrm{~mm}$, we were able to compress the plasma by more than a factor of 100 to a density of $2.6 \pm 0.6 \times 10^{10} \mathrm{~cm}^{-3}$. This number is a factor of 6 higher than the previous maximum positron density of $4 \times 10^{9} \mathrm{~cm}^{-3}$ [25]. In that case, the plasma contained only a couple of thousand particles, while we have compressed $20 \times 10^{6}$ positrons. The plasma aspect ratio after compression was more than 100 and the radius was less than $15 \mu \mathrm{m}$, while the length had increased by about $50 \%$, but was still well within the harmonic region of the trap. The density reported here is very close to the torquebalanced density found recently for electrons [26]. The torque-balanced upper limit on the density using a magnetic field of $3 \mathrm{~T}$ and a RW frequency of $15 \mathrm{MHz}$ is $3.1 \times$ $10^{10} \mathrm{~cm}^{-3}$. The observation that the compression rises uniformly as a function of frequency without showing signs of resonances associated with Trivelpiece-Gould modes also indicates that we are in the torque-balanced regime. There is no indication that we have reached a limit to the density using a RW, and it should thus be possible to achieve higher densities in the future using higher RW frequencies.

This work was supported in part by MEXT and RIKEN (Japan), CNPq (Brazil), SNF (Denmark), INFN (Italy), and the EPSRC (U.K.).

*Corresponding author.

Electronic address: lars.varming.jorgensen@cern.ch

†Electronic address: http://athena.web.cern.ch/athena/
[1] C. M. Surko and R. G. Greaves, Phys. Plasmas 11, 2333 (2004).

[2] A. P. Mills, Jr., Nucl. Instrum. Methods Phys. Res., Sect. B 192, 107 (2002).

[3] J. Clarke et al., in Non-Neutral Plasma Physics V, edited by M. Schauer, T. Mitchell, and R. Nebel, in AIP Conf. Proc. No. 692 (AIP, New York, 2003), p. 178.

[4] N. Oshima et al., Nucl. Instrum. Methods Phys. Res., Sect. B 205, 178 (2003).

[5] J.R. Danielson et al., in Non-Neutral Plasma Physics V (Ref. [3]), p. 149.

[6] C. M. Surko and R. G. Greaves, Radiat. Phys. Chem. 68, 419 (2003).

[7] M. Amoretti et al., Nature (London) 419, 456 (2002).

[8] G. Gabrielse et al., Phys. Rev. Lett. 89, 213401 (2002).

[9] G. Gabrielse et al., Phys. Lett. A 129, 38 (1988).

[10] M. H. Holzscheiter, M. Charlton, and M. M. Nieto, Phys. Rep. 402, 1 (2004).

[11] T. J. Murphy and C. M. Surko, Phys. Rev. A 46, 5696 (1992).

[12] L. V. Jørgensen et al., in Non-Neutral Plasma Physics IV, edited by F. Anderegg, L. Schweikhard, and C. F. Driscoll, in AIP Conf. Proc. No. 606 (AIP, New York, 2002), p. 35.

[13] M. Amoretti et al., Nucl. Instrum. Methods Phys. Res., Sect. A 518, 679 (2004).

[14] X.-P. Huang et al., Phys. Rev. Lett. 78, 875 (1997).

[15] R. G. Greaves and C. M. Surko, Phys. Rev. Lett. 85, 1883 (2000).

[16] M. Amoretti et al., Phys. Rev. Lett. 91, 55001 (2003).

[17] M. Amoretti et al., Phys. Plasmas 10, 3056 (2003).

[18] C. Regenfus, Nucl. Instrum. Methods Phys. Res., Sect. A 501, 65 (2003).

[19] N. Oshima et al., Phys. Rev. Lett. 93, 195001 (2004).

[20] J. Estrada et al., Phys. Rev. Lett. 84, 859 (2000).

[21] L. H. Haarsma et al., Phys. Rev. Lett. 75, 806 (1995).

[22] R.G. Greaves, M.D. Tinkle, and C.M. Surko, Phys. Plasmas 1, 1439 (1994).

[23] R. G. Greaves and J. Moxom, in Non-Neutral Plasma Physics V (Ref. [3]), p. 140.

[24] C. M. Surko, S. J. Gilbert, and R. G. Greaves, in NonNeutral Plasma Physics III, edited by J.J. Bollinger, 
R. L. Spencer, and R.C. Davidson, in AIP Conf. Proc. No. 498 (AIP, New York, 1999), p. 3.

[25] B. M. Jelenković et al., Phys. Rev. A 67, 063406 (2003).
[26] J.R. Danielson and C.M. Surko, Phys. Rev. Lett. 94, 035001 (2005). 